\title{
Pericytes Derived from Adipose-Derived Stem Cells Protect against Retinal Vasculopathy
}

\author{
Thomas A. Mendel ${ }^{1,2}$, Erin B. D. Clabough ${ }^{1 \times a}$, David S. Kao ${ }^{1}$, Tatiana N. Demidova-Rice ${ }^{3 \times b}$, \\ Jennifer T. Durham ${ }^{3}$, Brendan C. Zotter ${ }^{1}$, Scott A. Seaman ${ }^{6}$, Stephen M. Cronk ${ }^{6}$, Elizabeth P. Rakoczy ${ }^{4}$, \\ Adam J. Katz ${ }^{5 \times c}$, Ira M. Herman ${ }^{3}$, Shayn M. Peirce ${ }^{6}$, Paul A. Yates ${ }^{1 *}$
}

1 Department of Ophthalmology, University of Virginia, Charlottesville, Virginia, United States of America, 2 Department of Pathology, University of Virginia, Charlottesville, Virginia, United States of America, 3 Department of Molecular Physiology and Pharmacology, Tufts University, Boston, Massachusetts, United States of America, 4 Center for Ophthalmology and Visual Sciences, Department of Molecular Ophthalmology, The University of Western Australia, Nedlands, Western Australia, Australia, $\mathbf{5}$ Department of Plastic Surgery, University of Virginia, Charlottesville, Virginia, United States of America, 6 Department of Biomedical Engineering, University of Virginia, Charlottesville, Virginia, United States of America

\begin{abstract}
Background: Retinal vasculopathies, including diabetic retinopathy (DR), threaten the vision of over 100 million people. Retinal pericytes are critical for microvascular control, supporting retinal endothelial cells via direct contact and paracrine mechanisms. With pericyte death or loss, endothelial dysfunction ensues, resulting in hypoxic insult, pathologic angiogenesis, and ultimately blindness. Adipose-derived stem cells (ASCs) differentiate into pericytes, suggesting they may be useful as a protective and regenerative cellular therapy for retinal vascular disease. In this study, we examine the ability of ASCs to differentiate into pericytes that can stabilize retinal vessels in multiple pre-clinical models of retinal vasculopathy.

Methodology/Principal Findings: We found that ASCs express pericyte-specific markers in vitro. When injected intravitreally into the murine eye subjected to oxygen-induced retinopathy (OIR), ASCs were capable of migrating to and integrating with the retinal vasculature. Integrated ASCs maintained marker expression and pericyte-like morphology in vivo for at least 2 months. ASCs injected after OIR vessel destabilization and ablation enhanced vessel regrowth (16\% reduction in avascular area). ASCs injected intravitreally before OIR vessel destabilization prevented retinal capillary dropout (53\% reduction). Treatment of ASCs with transforming growth factor beta (TGF- $\beta 1$ ) enhanced hASC pericyte function, in a manner similar to native retinal pericytes, with increased marker expression of smooth muscle actin, cellular contractility, endothelial stabilization, and microvascular protection in OIR. Finally, injected ASCs prevented capillary loss in the diabetic retinopathic Akimba mouse (79\% reduction 2 months after injection).
\end{abstract}

Conclusions/Significance: ASC-derived pericytes can integrate with retinal vasculature, adopting both pericyte morphology and marker expression, and provide functional vascular protection in multiple murine models of retinal vasculopathy. The pericyte phenotype demonstrated by ASCs is enhanced with TGF- $\beta 1$ treatment, as seen with native retinal pericytes. ASCs may represent an innovative cellular therapy for protection against and repair of DR and other retinal vascular diseases.

Citation: Mendel TA, Clabough EBD, Kao DS, Demidova-Rice TN, Durham JT, et al. (2013) Pericytes Derived from Adipose-Derived Stem Cells Protect against Retinal Vasculopathy. PLoS ONE 8(5): e65691. doi:10.1371/journal.pone.0065691

Editor: Alexander V. Ljubimov, Cedars-Sinai Medical Center, United States of America

Received November 14, 2012; Accepted April 26, 2013; Published May 31, 2013

Copyright: (c) 2013 Mendel et al. This is an open-access article distributed under the terms of the Creative Commons Attribution License, which permits unrestricted use, distribution, and reproduction in any medium, provided the original author and source are credited.

Funding: This work was supported by Launchpad Translational Research Fund for Biomedical Innovation in Diabetes, Richmond Eye and Ear Foundation, National Institutes of Health (NIH) T32GM08715 to TAM, NIH HL082838-02 to SMP, and NIH K08 EY019533-02 to PAY. The funders had no role in study design, data collection and analysis, decision to publish, or preparation of the manuscript.

Competing Interests: The authors have declared that no competing interests exist.

*E-mail: pay2x@virginia.edu

aa Current address: Department of Biology, Randolph-Macon College, Ashland, Virginia, United States of America

ab Current address: E.L. Steele Laboratory for Tumor Biology, Massachusetts General Hospital, Harvard Medical School, Boston, Massachusetts, United States of America

ac Current address: Department of Plastic Surgery, University of Florida, Gainesville, Florida, United States of America

\section{Introduction}

Aberrant retinal angiogenesis and vasculopathies, including retinopathy of prematurity $(\mathrm{ROP})$, exudative age-related macular degeneration (AMD), and $\mathrm{DR}$, are among the leading causes of irreversible vision loss throughout the world [1]. DR alone afflicts an estimated 101 million people worldwide, with a prevalence of 155 million expected by 2030 [2]. While the pathogenesis and timing that underlie each condition differ, each involves destabilization of the retinal microvasculature.

Retinal pericytes, the cells that ensheath the retinal microvasculature, play a key role in the stabilization of endothelial cells, protecting them from hypoxic insults and angiogenic stimuli [3]. They are likely to be amongst the first cellular responders to diabetes-induced complications, a direct consequence of chronic diabetic inflammation [4,5]. Inadequate pericyte coverage of microvasculature has also been implicated in the pathogenesis of 
ROP [6,7]. Rich in actin [8], contractile pericytes are considered critical for microvascular control throughout the human body, supporting endothelial cells via contact-dependent [9] and soluble mediator-driven mechanisms [10,11]. Following pericyte death or loss, endothelial dysfunction results in downstream hypoxic insult, increased vascular endothelial growth factor (VEGF) production, macular edema, pathologic angiogenesis, and ultimately blindness [12].

Previous work has elegantly demonstrated that intravitreallyinjected bone marrow-derived stem cells (BMSCs) can help ameliorate and repair retinopathic insults. However, BMSCs appear to act primarily through their incorporation into the retina as endothelial cells, microglia, and photoreceptors $[13,14,15,16,17]$. Although pericytes can be derived from BMSGs [18], this does not appear to be a predominant differentiation pathway for these cells when injected into the eye [14,19].

ASCs are an alternative type of adult mesenchymal stem cells that are readily isolated from subcutaneous fat and contain a vascular progenitor subpopulation that is easily expanded for use in regenerative medicine [20]. Importantly, these cells are postulated to have a direct role in providing microvascular support and appear to differentiate readily as pericytes [20,21]. These attributes of ASCs suggest they could be useful for treatment of retinal microvascular disease.

To examine their potential therapeutic application in retinal vasculopathy, we sought to determine whether ASCs, once injected intravitreally, are able to migrate to and integrate with the retinal microvasculature. We also assessed the extent to which ASCs could replicate characteristics of endogenous retinal pericytes in in vitro assays of retinal pericyte function. We hypothesized that, as pericyte progenitors, ASCs could enable microvascular stabilization and offer protection against retinal vascular insults, including diabetic retinopathy.

\section{Materials and Methods}

\section{Cell Harvest}

Human adipose derived stem cells (hASGs) were obtained from patients under full approval of University of Virginia's Institutional Review Board. Adipose tissue was removed surgically from patients during elective plastic surgery by Adam Katz, MD, in the operating room at the University of Virginia, as previously described [22]. To prepare hASCs for in vitro and in vivo assays, the stromal vascular fraction was isolated from patient-derived lipoaspirate and further purified with serial passaging and subculturing, as we and others have previously shown [22]. Human bone marrow derived stem cells (hBMSCs) were purchased from Allcells (\#MSC-001F). Bovine retinae and retinal microvascular endothelial cells (BRECs) and pericytes were isolated and characterized as previously described $[8,23,24,25]$. The tissues were received from an abattoir and no institutional animal use committee approval was required. Mouse adipose derived stem cells (mASCs) were purchased from Eton Bioscience (280004001G) for functional studies or isolated from the epididymal fat pads of 9-week old Akita mice for integration studies $[26,27]$. The epididymal fat pads were digested in collagenasecontaining digestion buffer $(0.01 \%$ collagenase type I, $2.5 \%$ BSA, $200 \mathrm{nM}$ adenosine, $20 \mathrm{mM}$ HEPES, $120 \mathrm{mM} \mathrm{NaCl}, 4.7 \mathrm{mM}$ $\mathrm{KCl}, 1.3 \mathrm{mM} \mathrm{CaCl}{ }_{2}-2 \mathrm{H}_{2} \mathrm{O}, 1.2 \mathrm{mM} \mathrm{KH}_{2} \mathrm{PO}_{4}, 1.2 \mathrm{mM} \mathrm{MgSO}_{4^{-}}$ $\left.7 \mathrm{H}_{2} \mathrm{O}\right)$ for one hour at $37 \mathrm{C}$. The resulting mixture was filtered through $200-\mu \mu$ mesh to exclude any undigested tissue. The filtrate was centrifuged to remove remaining collagenase, and the pellet (containing cells) incubated with red blood cell lysis buffer (15.5 mM NH $4 \mathrm{Cl}, 10 \mathrm{mM} \mathrm{KHCO}_{3}, 0.1 \mathrm{mM}$ EDTA) for 5 minutes at room temperature to lyse red blood cells. The cell suspension was then sterile-filtered through $40-\mu \mu$ mesh and plated on sterile culture plates.

\section{Cell Culture}

Isolated hASGs and mASCs were cultured in $10 \mathrm{~cm}$ Nunc culture dishes (Thermoscientific \#12-565-020) using Gibco DMEM/F12 (1:1) (Life Technologies \#11320-033), supplemented with 10\% Gibco fetal bovine serum (Invitrogen \#16000044, Lot \#953873), 1\% antibiotic-antimycotic (Invitrogen \#15240062). hBMSCs were cultured in the same media as hASCs, but augmented to $20 \%$ fetal bovine serum. Media was replaced every three days and cells were passaged at $70 \%$ confluency using Stempro Accutase (Gibco/Life Technologies \#A11105-01). Cells were maintained at $37^{\circ} \mathrm{C}, 5 \% \mathrm{CO}_{2}$, and $75 \%$ humidity. hASCs and mASCs were used from passage 3 to 6 in all studies. hBMSCs were used at passage 3 . Bovine retinae and retinal microvascular endothelial cells and pericytes were isolated and characterized as previously described $[8,25]$.

\section{Cellular Contractility Assay}

The assay was performed essentially as described [28]. Briefly, round $12 \mathrm{~mm}$ diameter cover slips were coated with a thin layer of silicone that was then thermally cross-linked. Coverslips were charged using a glow discharge apparatus and were coated with Type I collagen suspended in PBS $(0.1 \mathrm{mg} / \mathrm{mL}$, BD Biosciences \#354236). The prepared cover slips were placed in the wells of 24well tissue culture plates, sterilized for 3 minutes with UV radiation, and seeded with $2 \times 10^{3} \mathrm{hASC}$ or bovine retinal pericytes. Cellular contraction caused deformation of the silicone substrate, which was visualized by brightfield imaging. 24 hours after plating, hASCs were either left in serum-containing media or treated with media containing $10 \mathrm{ng} / \mathrm{mL}$ recombinant human TGF- $\beta 1$ (rhTGF- $\beta 1$, R\&D Systems \#240B). Following another 24 hour incubation period, control or rhTGF- $\beta 1$-treated hASCs were visualized with an Axiovert $200 \mathrm{M}$ microscope (Carl Zeiss MicroImaging, Thornwood, NY) using $5 \times$ or $10 \times$ objective lenses $[25,28]$. In order to quantify changes in hASG contractility induced by rhTGF- $\beta 1$, we determined the percentage of wrinkling cells cultured in the presence or absence of $\operatorname{rhTGF} \beta-1$. The values were recorded using Microsoft Excel and are shown as a mean $+/-\mathrm{SD}$.

\section{Co-Culture Assay}

This assay was performed as described previously [25] with the following modifications. UV-sterilized round cover slips were placed into the wells of 24-well tissue culture plates and seeded with either bovine retinal pericytes or hASGs at a density of $3 \times 10^{3}$ cells/well. After the cells adhered overnight, cultures were either incubated with normal $10 \%$ serum-containing growth media or pre-conditioned with $1 \mathrm{ng} / \mathrm{mL}$ rhTGF- $\beta 1$ in $0.5 \%-5 \%$ serumcontaining media for 24-48 hours. Pericyte and hASG cultures were washed with PBS to remove the rhTGF $\beta-1$, and BRECs were added to the wells at a density of $5 \times 10^{3}$ cells/well in $5 \%$ serum-containing media. For control experiments, BRECs were plated in monoculture, and allowed to attach overnight. For BREC monoculture, half the wells were treated with $5 \%$ serum media while the remainder was treated with $1 \mathrm{ng} / \mathrm{mL}$ rhTGF- $\beta 1$ in 5\% serum-containing media. Both mono- and co-cultures were incubated for 24 hours, then S-phase entry (endothelial proliferation) was assessed using the Click-i ${ }^{\circledR}$ EdU Cell Proliferation Kit (Invitrogen \#C10339) according to the manufacturer's instructions. Following EdU detection, hASG and pericyte cultures were stained using mouse anti-smooth muscle actin (SMA) (Biogenex 
\#MU128-UG) and anti-mouse Alexa-Fluor 488 secondary antibody (Invitrogen A-21200). Fluorescence and phase contrast imaging was performed following fixation and staining as previously described [25]. Endothelial cell S-phase entry in control and treated co-cultures both for BREGs that were isolated or those that were contacting either hASCs or bovine retinal pericytes was then quantified and values expressed +/-SD.

\section{In Vitro Immunohistochemistry}

hASCs and mASCs were briefly washed in PBS, fixed using methanol $\left(10 \mathrm{~min}\right.$ at $\left.-20^{\circ} \mathrm{C}\right)$, rinsed with PBS $(3 \times 5 \mathrm{~min})$, blocked with $1 \%$ BSA, $2 \%$ goat serum, $0.1 \%$ Triton $\mathrm{X}$ in PBS, exposed to primary antibodies in blocking solution $\left(4^{\circ} \mathrm{C}\right.$ overnight), washed with PBS $(3 \times 10 \mathrm{~min})$, and either cover slipped or exposed to secondary antibody (45 min), followed by washing with PBS $(3 \times 10 \mathrm{~min})$. Antibodies used for hASCis were mouse monoclonal SMA-FITC (Sigma-Aldrich F3777; 1:250), rabbit polyclonal NG2 (Millipore AB5320; 1:100), rabbit polyclonal PDGFR $\beta$ (Santa Cruz sc-432; 1:100), and goat anti rabbit Cy2 secondary (Millipore; 1:250). mASGs were stained with anti-SMA preconjugated to Cy3 (Sigma \#C6198-.2ML).

\section{Flow Cytometry}

Antibody characterization of hASCs (passage 4) was performed on a Becton Dickinson/Cytek FACSCalibur G with CellQuest Pro acquisition software, using FlowJo software for analysis. Cells were lifted using Accutase (20 min), fixed with methanol $\left(10 \mathrm{~min}\right.$ at $\left.-20^{\circ} \mathrm{C}\right)$, washed with $1 \%$ BSA in PBS, and permeabilized with $0.1 \%$ Triton $\mathrm{X}, 1 \%$ BSA in PBS $\left(10 \mathrm{~min}\right.$ at $\left.4^{\circ} \mathrm{C}\right)$. Primary antibodies were incubated in $1 \%$ BSA in PBS (45 min), washed with $1 \%$ BSA in PBS $(2 \times 10 \mathrm{~min})$, and re-suspended in $500 \mu \mathrm{L} 1 \%$ BSA in PBS for analysis. Antibodies used for hASGs were mouse monoclonal $\beta$-actin-FITC (Abcam ab64496), mouse monoclonal SMA-FITC (Sigma-Aldrich F3022; 1:500), rabbit polyclonal PDGFR $\beta$ (Santa Cruz sc-432; 1:200), mouse IgG1-FITC isotype control (BD Pharmingen 554679; $0.5 \mathrm{ug} / \mathrm{uL})$, and goat anti rabbit Cy2 secondary (Millipore; 1:250). Results were replicated using at least 3 separate experiments. mASCs (passage 4) were evaluated on a BD FacsCanto II with $405 \mathrm{~nm}, 488 \mathrm{~nm}$ and $633 \mathrm{~nm}$ lasers. mASCs were stained with PDGFR $\beta$ (CD140b) preconjugated to APC (eBioscience \#17-1402-80, 1:200), $\alpha$-smooth muscle actin preconjugated to Cy3 (Sigma \#C6198-.2ML, 1:200).

\section{Ocular Injections}

Mice were anesthetized with ketamine/xylazine injected intraperitoneally or with inhaled isoflurane. If palpebral fissures were not yet open, they were carefully cut with iris scissors, followed by local application of proparacaine. Prior to injection, hASCs, mASCs, or hBMSGs were stained with 1 ,'-Dioctadecyl-3,3,3',3'tetramethylindocarbocyanine perchlorate (DiI, Invitrogen D282), using a standard labeling technique for tracking of adult mesenchymal stem cells [29,30,31,32,33,34]. hASCs, mASCs, or hBMSCs were suspended in 0.5 to $1.5 \mathrm{uL}$ of PBS and were injected into the vitreous of the study eye with a 33 gauge Hamilton needle through the pars plana. An equal volume of PBS was injected into the contralateral eye to serve as a paired control. For palpebral fissures cut with iris scissors, a small amount of methylcyanoacrylate was used to re-seal the lids. All injected ASCs were passage 4 to passage 6 at the time of injection. BMSCs were passage 3 at the time of injection.

\section{Oxygen Induced Retinopathy (OIR)}

All procedures performed with mice conformed to the guidelines within the ARVO Statement for the Use of Animals in Ophthalmology and Vision Research and were approved by the University of Virginia's Animal Care and Use Committee. NOD SCID mice (Charles River) were immersed in 94\% oxygen from postnatal day 7 (P7) to day 12 (Biospherix A30274-P, P-1 10-E702), as $75 \%$ oxygen did not produce substantial central avascular area in NOD SCID pups. The mice were checked through the clear plastic door twice daily for the duration of the oxygen treatment. Retinas were harvested as described below and retinal wholemounts prepared. Areas of capillary pruning (major vessels remain intact) were traced manually by blinded individuals and quantified in ImageJ. Following OIR, 10,000 hASCs in $1.5 \mu \mathrm{L}$ of PBS were injected into the vitreous of the $\mathrm{P} 12$ eye, with an equal volume of PBS alone injected into the contralateral control eye. As a cell type control for the OIR recovery model, two litters ( $\mathrm{n}=10 \mathrm{P} 12 \mathrm{pups}$ ) of NOD SCID mice were used with either 10,000 hBMSCs or 10,000 hASCs in $1.5 \mu \mathrm{L}$ of PBS injected into the vitreous of the $\mathrm{P} 12$ eye, and $1.5 \mu \mathrm{L}$ of PBS alone in the contralateral control eye. To account for litter variability, half the mice in these litters received hBMSCs ( $n=5$ eyes of 5 P12 pups) in the study eye, while the other half received hASCs ( $n=5$ eyes of 5 P12 pups) in the study eye. Use of two NOD SCID litters controlled for known litter and littermate variability. All eyes were harvested and examined for areas of capillary pruning at P14. For the OIR protection model, 600 hASGs suspended in $0.5 \mu \mathrm{L}$ of PBS were injected at P2 into the study eye and $0.5 \mu \mathrm{L}$ of PBS alone in the contralateral control eye. Eyes were harvested and examined for areas of capillary pruning at P12 following OIR. For the rhTGF$\beta 1$ OIR protection model, 1000 hASCs were suspended in $0.5 \mu \mathrm{L}$ of PBS after 48 hours of rhTGF- $\beta 1$ pretreatment and subsequent media replacement. They were then injected at P6 into one eye and 1000 untreated hASCs suspended in $0.5 \mu \mathrm{L}$ of PBS were injected into in the contralateral eye. Eyes were harvested and examined for areas of capillary pruning at P12 following OIR.

\section{Akimba Model of Diabetic Retinopathy}

Kimba and Akita mice were obtained from Elizabeth Rakoczy, $\mathrm{PhD}$, and crossed as previously described to establish a breeding colony for further ASC studies [35]. For functional microvascular studies, intraocular injections for the Akimba vascular protection model were performed at P9 with mASCs (Eton Bioscience, passage 3) cultured as described (Cell Harvest, Ocular Injections). Specifically $1,000 \mathrm{mASC}$ in $0.5 \mu \mathrm{L}$ of PBS were injected into the vitreous of the P9 eye, with equal volume of PBS alone in the contralateral eye. Two months after injection, fluorescein angiography was performed by intraperitoneal injection of $0.1 \mathrm{~mL}$ of $10 \%$ fluorescein reconstituted in PBS into mice anesthetized with Ketamine/Xylazine. A Cantor Nissel contact lens was applied to the cornea and the retinal vasculature imaged on a Heidelberg Spectralis retinal imager as previously described. Following angiography, retinas were harvested and retinal wholemounts prepared as described below in the Retinal Wholemounts section of Methods. Areas of capillary dropout, as defined by areas that have lost tertiary retinal branches of retinal microvasculature, were circumscribed by blinded observers and recorded in ImageJ. In a second experiment we examined the potential for homologous incorporation of mASCs at late time points. For this latter experiment, mASCs were harvested from Akimba mouse epididymus, cultured, and then subsequently injected (as described above in Cell Harvest section of Methods). Specifically 10,000 mASCs in $1.5 \mu \mathrm{L}$ of PBS were injected into the vitreous of the 5 week old Akimba eye $(n=8$ eyes of 8 mice), with equal volume of 
PBS alone in the contralateral eye. Retinas were harvested one month later at 9 weeks of age and wholemounts prepared (as described below in the Retinal Wholemounts section of Methods). Retinal vessels were lectin and SMA stained, and imaged for incorporation of DiI-labeled mASCs into the retina and association with the retinal microvasculature.

\section{Retinal Wholemounts}

Mice were sacrificed by overdose of ketamine/xylazine injected intraperitoneally and then perfused for 5 minutes with $4 \%$ paraformaldehyde by insertion of a needle into the left ventricle of the heart, attached to a perfusion pump, following cutting of the inferior vena cava. Eyes were then enucleated and immersed in $4 \%$ paraformaldehyde for 10 minutes, without any perforation of eye, before being transferred to PBS at room temperature. Cornea, extraocular muscles, optic nerve, iris, sclera, retinal pigment epithelium, lens, and hyaloid vasculature were then carefully removed in that order. Four relaxing cuts were made to enable flatmounting on a gelatin-coated slide. After permeabilization with $1 \mathrm{mg} / \mathrm{mL}$ digitonin (MP Biomedicals 0215948050) for 1 hour, retinas were stained with mouse monoclonal SMA-FITC (Sigma-Aldrich F3022; 1:250), isolectin IB4-647 (Invitrogen I32450; 1:200), isolectin IB4-488 (Invitrogen I21411; 1:200), anti-NG2 chondroitin sulfate proteoglycan (Millipore AB5320; 1:100), and goat anti-rabbit IgG Cy2-conjugated (Millipore AP132J; 1:250). Slides were mounted using fluorogel with TRIS buffer (Electron Microscopy Sciences 17985-10).

\section{Data Quantification and Statistics}

Retinas were imaged using a Leica TCS SP2 confocal with DMIRE2 inverted microscope and a Zeiss LSM 510 confocal microscope. $10 \times$ images were montaged using i2kAlignRetina (Dual Align, LLC) or the MosaicJ plugin in the Fiji Software version of ImageJ. Figures were constructed in Adobe Photoshop CS5. Except where indicated, in order to be conservative with statements of statistical significance in light of non-Gaussian dissimilar variance, comparisons were made using median values and significance tested with nonparametric Wilcoxan Matched Pairs and Mann-Whitney tests, calculated on GraphPad Prism (version 6.00 for Macintosh, GraphPad Software, La Jolla California USA, www.graphpad.com). Error bars represent standard deviation. Statistical significance is denoted in all figures in the manuscript by asterisks with * for $\mathrm{p} \leq 0.05$, and $* *$ for $\leq 0.01$, and $* * *$ for $\leq 0.001$.

\section{Results and Discussion}

\section{Retinal Incorporation of Intravitreally Injected hASCs}

We initially sought to determine if ASCs could be efficiently delivered to the retina through intravitreal pars plana injection, and once there, whether they would successfully integrate with the retinal vasculature. We injected hASCs intravitreally, similar to previously published BMSG retinal incorporation experiments [13]. In particular, our goal was to assess the ability of intravitreally delivered ASCs to assume abluminal locations around retinal capillaries, which is a defining characteristic of pericytes.

In the OIR model, the central retinal microvasculature is ablated during a hyperoxic insult that takes place from P7 to P12 [12], while the major vessels remain. Revascularization of the central retina, including recruitment of native retinal pericytes, occurs once mice are returned to room air at P12 [36]. hASGs suitable for injection were derived from human lipoaspirates that were serially passaged in culture, due to the known enrichment of a pericyte-like phenotype at later passages [37]. Before injection, hASCs were labeled with DiI, as used by others for tracking adult mesenchymal stem cells in vivo [29,30,31,32,33,34]. Initial injections with 10,000 DiI-labeled hASGs suspended in sterile PBS were performed through the pars plana at P12 after mice had first been exposed to OIR [14,38,39,40,41].

hASGis were observed in abundance alongside central retinal microvasculature 10 days after injection (Fig. 1A). 8 weeks after injection, $308 \mathrm{hASC}$, or $3.08 \%(2.08-4.07 \%$ 95\% CI) of the 10,000 injected cells, remained engrafted, which is comparable to stem cell engraftment efficiencies observed in other model systems [42]. $85.6 \%$ (82.8-88.7\% 95\% CI) of engrafted hASCs were found in physical contact with retinal microvessels, many adopting pericyte-like abluminal locations and phenotypic wrapping around vessels (Fig. 1B, G). While our studies to date do not conclusively rule out some proliferation of injected ASCs after injection into the eye, the numbers of cells observed two months after injection were not qualitatively more than the number of cells in eyes harvested one week after injection.

\section{Verification of hASC-derived Pericyte Marker Expression in vitro and in vivo}

While a functional pericyte phenotype for hASCs is suggested by their morphology and perivascular location, we sought to explore hASC expression of characteristic pericyte markers. Alpha smooth muscle actin (SMA), nerve/glial antigen 2 (NG2), and platelet derived growth factor receptor beta (PDGFR $\beta$ ) frequently designate a pericyte phenotype, although specific isoprotein expression is dependent upon microvascular context in vivo $[43,44]$. We found that hASCs express abundant steady state protein levels characteristic of native microvascular pericytes, including SMA, NG2, and PDGFR $\beta$ as measured by both antibody staining and flow cytometry (Fig. 1D-I). 91.2\% of hASCs tested co-expressed both SMA and PDGFR $\beta$ (Fig. 1J). Additionally, hASCs injected intravitreally into NOD SCID mice at P12, following OIR induction, also continue to express SMA and NG2, when harvested six to eight weeks post-injection (Fig. 1K-L). Both our in vivo and in vitro studies indicate that serially passaged hASCs can differentiate into retinal pericytes in response to microenvironmental cues.

Importantly, no ocular tumor formation was observed and no metastatic lesions in the lung or liver were found at these time points, consistent with prior reports on in vivo hASG use [45]. Sagittal sections of hASC injected eyes stained with either Oil Red $\mathrm{O}$ or Alcian Blue did not reveal any off target differentiation of hASCs into adipose or chondrocyte lineages (data not shown). Similarly, antibody staining with F4/80, a microglial and macrophage marker, did not show any definitive differentiation into these cell types. Interestingly, we saw no significant cell trafficking to deeper layers of the retina on sagittal sections or 3D confocal reconstruction of the retinal vasculature. Sub-retinal hASCs were sometimes seen near the site of injection, often associated with a localized retinal detachment, suggesting that trafficking to these locations occurred at the time of injection.

\section{hASCs Accelerate Recovery from Oxygen Induced Retinopathy}

Having demonstrated that hASCs could home to and integrate with retinal vasculature with stable expression of pericyte markers, we next assessed whether these injected hASCs would functionally affect revascularization of the central retina following OIR injury. As before, eyes were injected with hASCs at P12 following OIR and then harvested at P14, which in NOD-SCID mice is the 
A

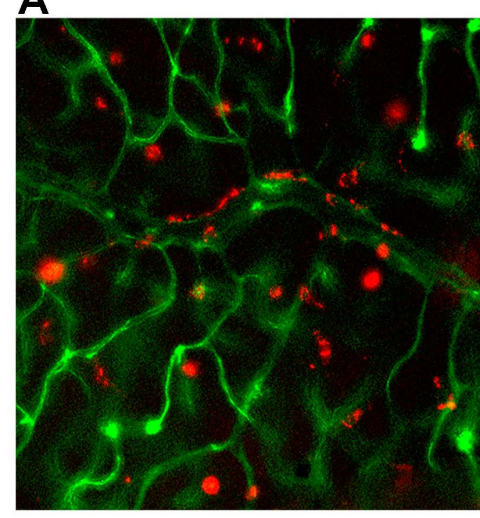

D

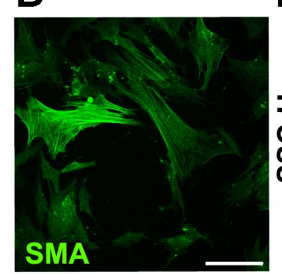

J
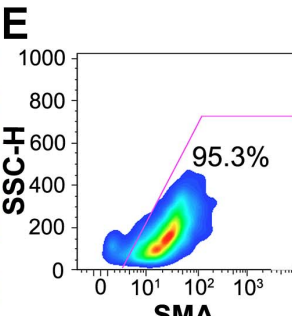

SMA

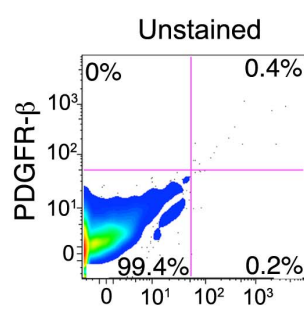

SMA

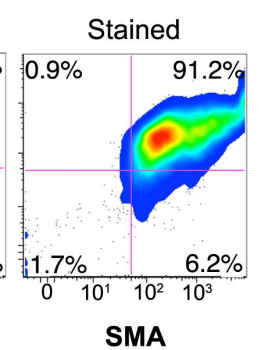

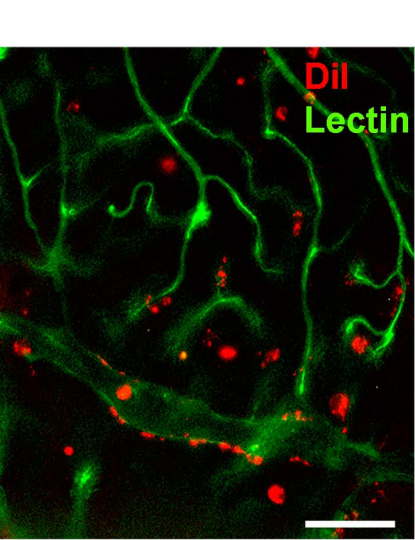

B

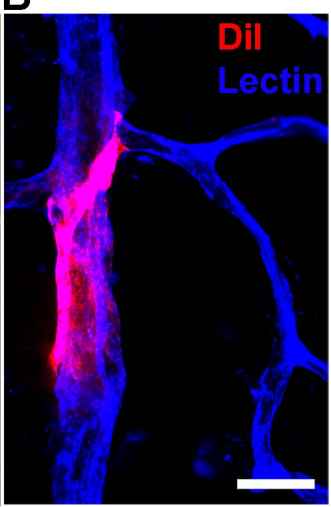

C

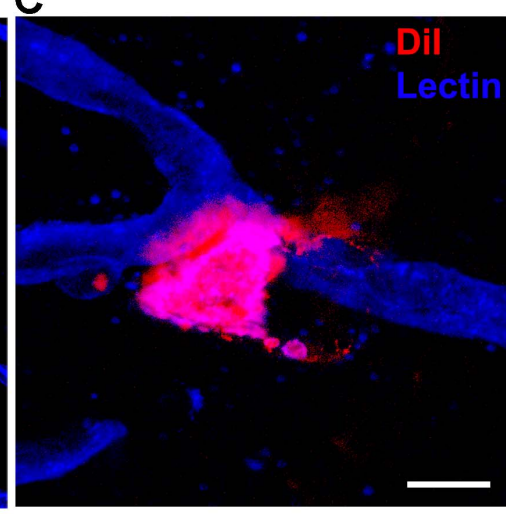

H

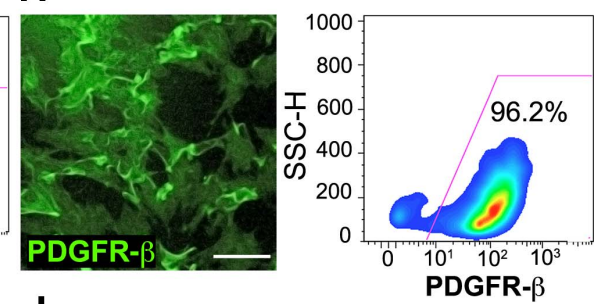

L

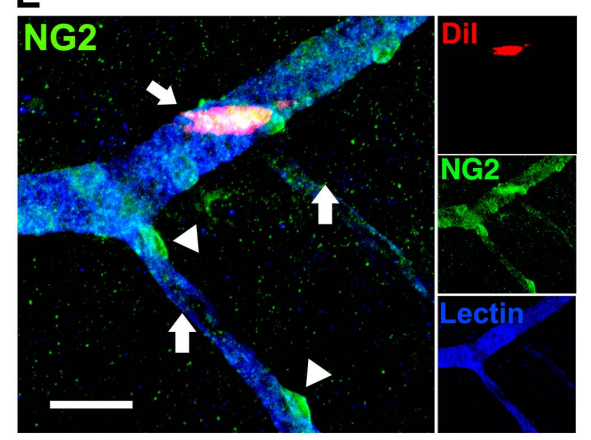

Figure 1. hASCs demonstrate pericyte-like morphology and phenotype markers in vitro and in vivo. A, Dil labeled (red) hASCs injected intravitreally at postnatal day 12 (P12) following oxygen induced retinopathy (OIR) home from the vitreous to murine retinal microvasculature (green) as seen following harvest at P22. B-C, Dil-labeled hASCs (red) wrap around isolectin labeled retinal microvessels (blue) abluminally and target vascular junctions, both properties of terminally differentiated pericytes. D-I, Passage $5 \mathrm{hASCs}$ exhibit in virto expression of the characteristic pericyte markers smooth muscle actin (SMA, D-E), nerve/glial antigen 2 (NG2, F-G), and platelet derived growth factor receptor beta (PDGFR- $\beta$, H-I) by both immumohistochemical staining on cultured cells and by flow cytometry on cells harvested from these cultures. J, $91.2 \%$ of hASCs demonstrated colabeling of SMA and PDGFR- $\beta$ compared to unstained controls. K-L, Dil labeled hASCs injected intravitreally into NOD SCID mice at P12, after OIR hyperoxia, maintain intimate association with the retinal microvasculature 6-8 weeks later and demonstrate persistent SMA (K), NG2 (L) (white arrows), with SMA extending into the cellular extension wrapping around the capillary. On average $308 \mathrm{hASCs}$, or $3.08 \%$ of the 10,000 injected cells, remained engrafted, with $85.6 \%$ of engrafted hASCs found in physical contact with retinal microvessels. Note native retinal pericytes are also labeled with NG2 (L) but lack Dil staining (white arrowheads). In contrast, no native retinal pericytes are seen labeled with SMA (K) given that this is a tertiary branch of the retinal vasculature, and native pericyte SMA expression is typically seen only on primary and secondary vessels. Scale bars: $\mathbf{A}=200 \mu \mathrm{m}$, $\mathbf{B}=20 \mu \mathrm{m}, \mathbf{C}=10 \mu \mathrm{m}, \mathbf{D}, \mathbf{F}, \mathbf{H}=100 \mu \mathrm{m}, \mathbf{K}=10 \mu \mathrm{m}, \mathbf{L}=20 \mu \mathrm{m}$.

doi:10.1371/journal.pone.0065691.g001

midpoint of post-OIR revascularization. Contralateral eyes were injected with an equal volume of PBS vehicle, serving as a commonly employed carrier control [14,46,47,48,49], and allowing paired comparison of eyes. NOD-SCID mice were chosen to avoid the potential immune rejection of our xenografted hASGs that might confound our analysis of revascularization. Flatmounted retina demonstrated a $16.4 \%$ reduction in avascular area $(\mathrm{n}=17, \mathrm{p}=0.03)$, indicating greater central retinal revascularization compared to contralateral control retinas injected with PBS (Fig. 2A-C). No appreciable pre-retinal neovascularization was observed at any time point in either control or hASC injected eyes, likely due to our use of immunocomprimised mice, since inflammatory stimuli have been shown to be a large component of the aberrant neovascular response [50].

We performed an additional experiment to benchmark hASCs against a cell-type control. Two NOD SCID litters ( $\mathrm{n}=10 \mathrm{pups}$ total) were injected with either 10,000 hASCs ( $n=5$ eyes of 5 pups) or 10,000 human bone marrow derived stem cells (hBMSCs) ( $\mathrm{n}=5$ eyes of 5 pups) in the study eye at P12, along with an equal volume of PBS in the contralateral control eye $(n=10$ eyes of 10 pups). Unsorted hBMSGs were chosen as a negative control cell since sorted lineage negative murine BMSCs are another mesenchymal stem cell previously shown to influence retinal vasculature [13]. Consistent with the earlier results of this study, 
A

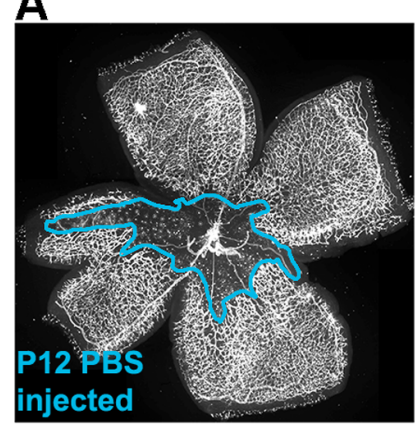

D

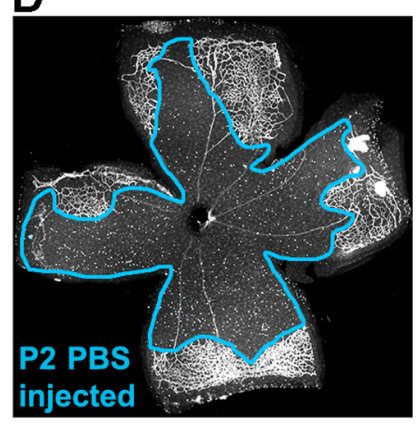

B

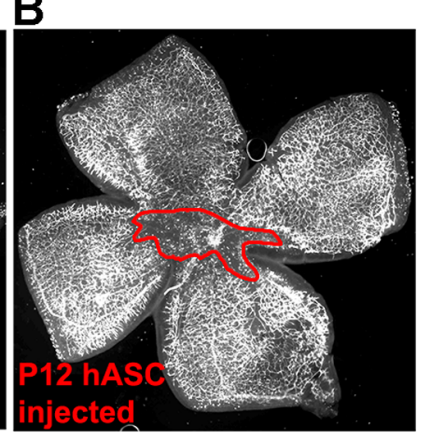

$\mathbf{E}$

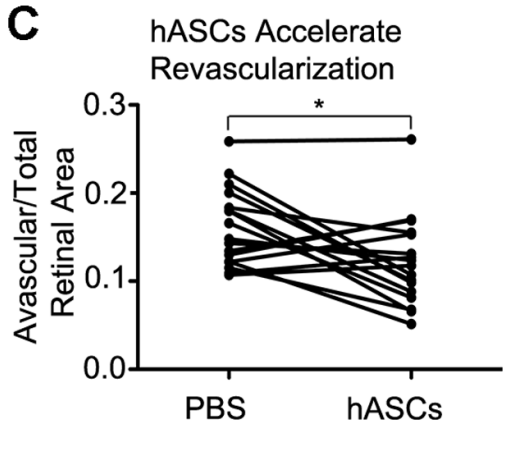

F hASCs Prevent Hyperoxia-

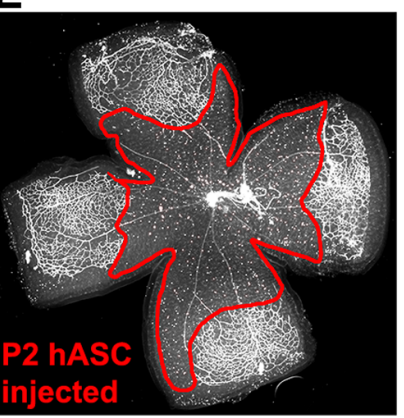

Induced Central Retinal Ablation

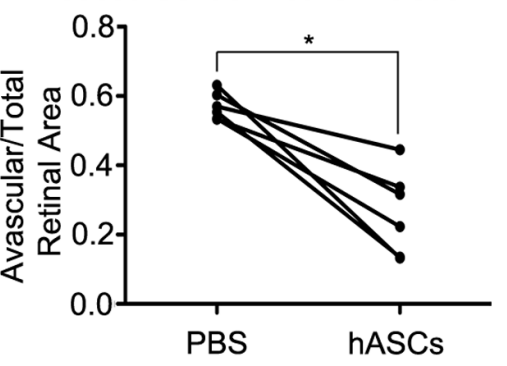

Figure 2. Human adipose-derived stem cells (hASCs) stabilize oxygen-induced retinopathy (OIR). A-C, Eyes of NOD SCID mice, injected intravitreally with hASCs at P12 following OIR, and then harvested at P14, demonstrate improved revascularization of central retina as compared to contralateral PBS injected (blue) carrier controls $(16.4 \%$ reduction, $n=17, p=0.03$ ). D-F, NOD SCID eyes injected intravitreally with $h A S C s$ at $P 2$, prior to OIR, exhibited dramatically less central vascular ablation at P12 than contralateral PBS injected carrier controls (blue) $(52.9 \%$ reduction, $\mathrm{n}=6$, $p=0.03$ ). Lines in $\mathbf{C}$ and $\mathbf{F}$ connect data points for hASC injected and contralateral PBS injected eyes in the same mouse.

doi:10.1371/journal.pone.0065691.g002

hASCs accelerate revascularization of the central retina by $16.4 \%$ at P14 compared to contralateral PBS injected control eyes $(\mathrm{n}=5$ pups, $\mathrm{p}=0.03)$. However, hBMSCs had no statistically significant effect on the rate of revascularization compared to contralateral PBS injected control eyes $(n=5$ pups, $\mathrm{p}=0.29)$ (Fig. S1).

\section{hASCs Protect against Oxygen Induced Retinopathy}

We next determined whether injected hASCs could protect central retinal microvessels against destabilization and ablation seen during OIR. Eyes were injected at P2 with 600 DiI labeled hASGs suspended in $0.5 \mu \mathrm{l}$ PBS due to the smaller ocular volume at $\mathrm{P}$ 2, with contralateral eyes receiving equal volumes of $\mathrm{PBS}$ as a vehicle control. Notably, injection of hASCs at P2 does not appear to appreciably affect normal retinal vascularization through $\mathrm{P} 7$ (Fig. S2). Injected mice underwent OIR from P7 to P12 per standard protocol [36] but with oxygen concentration elevated to $94 \%$ required for NOD SCID central retinal microvascular ablation. Retinal wholemounts at P12 demonstrated a profound $52.9 \%$ reduction of avascular area $(n=6, p=0.03)$ in hASGtreated eyes as compared to contralateral PBS-injected controls (Fig. 2D-F).

This result suggests that injected hASCs may assist in stabilizing retinal microvasculature that is otherwise acutely unstable. Although the mechanism underlying this protection is not yet clear, we have found that injected hASCs efficiently integrate into the retinal microvasculature after P8 and we observe a $57 \%$ increase $(\mathrm{n}=16$ and $12, \mathrm{p}=0.031)$ in vascular length density in retinal sub-fields containing labeled cells as compared to sub-fields without cells. This finding is consistent with prior studies demonstrating decreased vessel susceptibility to OIR correspond- ing to an increased desmin ensheathment ratio, which serves as a surrogate for pericyte coverage [6,7].

However, this explanation seems insufficient to account fully for the observed results. Few cells are seen integrated with the retinal microvasculature at $\mathrm{P} 7$, prior to OIR, with less than $5 \%$ of total injected cells found associated with the microvasculature by $\mathrm{P} 12$, following OIR. Given that ASGs are known to secrete a large repertoire of trophic factors and that the majority of injected cells remain in the vitreous upon dissection, its seems likely that hASCs mediate protection of retinal microvessels at least in part via paracrine signaling mechanisms.

\section{hASCs Respond to Pre-treatment with TGF- $\beta 1$ in a Manner Analogous to Endogenous Retinal Pericytes}

TGF- $\beta 1$ has been previously demonstrated to have a direct effect on retinal pericytes and influence their potential interactions with endothelial cells. Its defined roles include helping maintain retinal vascular barrier function [51], increasing expression of smooth muscle actin [52], and enhancing pericyte contraction [53], which have been suggested to help stabilize endothelial cells $[54,55]$. We wished to evaluate the extent to which hASCpericytes might also be influenced by TGF- $\beta 1$ pre-treatment, if these treatment effects were analogous those observed with retinal pericytes, and if TGF- $\beta 1$ might enhance hASC functionality within in vitro and in vivo assays of pericyte function and vascular stabilization. We first performed an hASG and bovine retinal endothelial cell (BREC) co-culture assay previously described for evaluating retinal pericytes [25]. Using this assay, we tested whether pre-conditioning the hASC and/or retinal pericytes with TGF- $\beta 1$ would enhance these cells' ability to inhibit BREC cell cycle entry in a contact-dependent manner. After 24-48 hours of 
pre-conditioning hASC or pericytes with TGF- $\beta 1$, we found that BRECs contacting the TGF- $\beta 1$ pre-treated hASCs are $14.4 \%$ less likely to be found in mitosis, as compared to co-cultures where endothelial cells contacted hASGs without TGF- $\beta 1$ pre-conditioning $(p<0.05)$. This result is comparable to the $15.7 \%$ decrease in endothelial S-phase entry in cells contacting pericytes $(p<0.05)$ (Fig. 3A, B). BRECs in monoculture demonstrate a $23.1 \%$ decrease $(\mathrm{p}<0.01)$ in mitotic activity after direct constant exposure to TGF- $\beta 1$ (Fig. 3A, B). Thus, pre-conditioned pericytes and hASCs contacting BREGs recapitulate most, but certainly not all, of the reduction in mitotic activity seen with direct TGF- $\beta 1$ application to BRECs.

Furthermore, hASCs pre-treated with TGF- $\beta 1$ demonstrated a 2.6 fold enhancement of contractility $(n=32$ treated cells, 40 untreated cells, $\mathrm{p}=0.0413$ ) over untreated hASCs, as measured by the cells' ability to deform an underlying silicone substratum (Fig. 3C, D). This is analogous to results obtained when comparing
TGF- $\beta 1$ and non-TGF- $\beta 1$ treated endogenous bovine retinal pericytes [53]. TGF- $\beta 1$ pre-treatment increased the fraction of hASCs highly expressing SMA by $15.3 \%$ without altering control $\beta$-actin levels (Fig. 3E, F), which may in part account for their enhanced contractility.

Having demonstrated in vitro effects of TGF- $\beta 1$ pre-treatment on hASCs, we next sought to determine whether this would functionally improve their performance in protecting retinal vasculature from the effects of OIR. P6 NOD SCID pups were intravitreally injected with either 1,000 untreated or 1,000 TGF$\beta 1$ pre-treated hASGs in $0.5 \mu \mathrm{L}$ PBS. When harvested at P12 after hyperoxia, eyes injected with TGF- $\beta 1$ pre-treated hASCs demonstrated an $11.0 \%$ reduction in avascular area $(n=22, p=0.01)$, compared to the contralateral eyes injected with untreated hASCs (Fig. 3G-I). This result suggests that TGF- $\beta 1$ pre-treatment enhances hASC stabilization of retinal vessels in vivo.
A

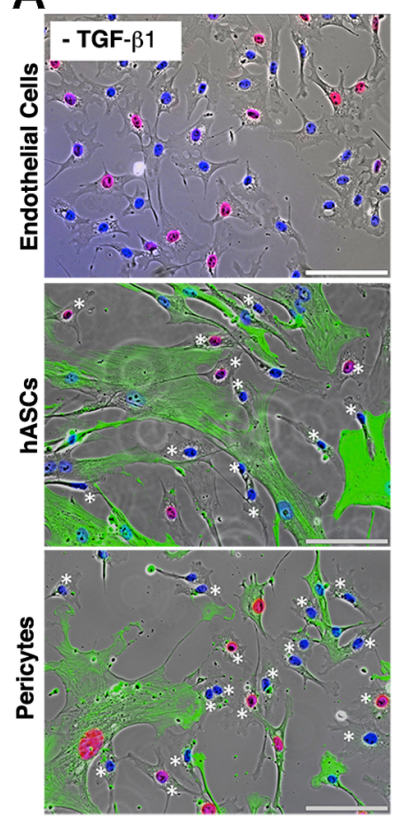

E

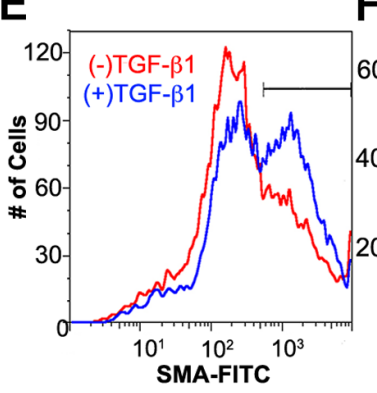

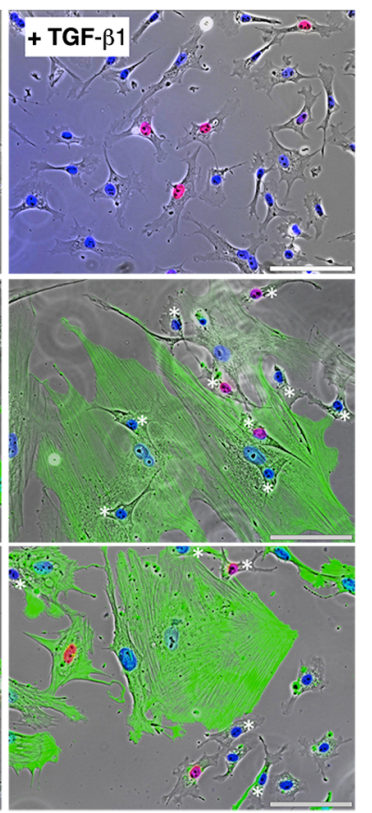

$\mathbf{F}$
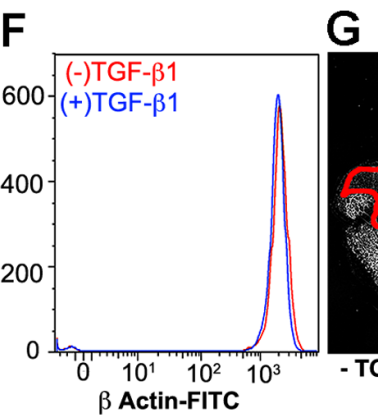

B

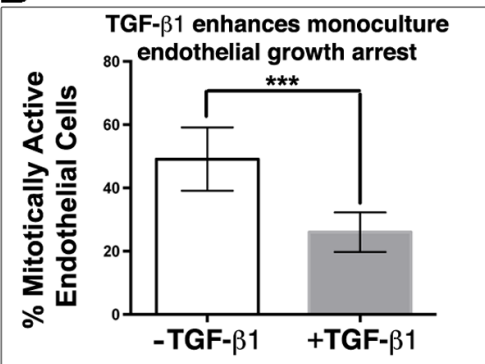

TGF- $\beta 1$ preconditioning of hASCs or pericytes enhances hASC contactdependent endothelial growth arrest
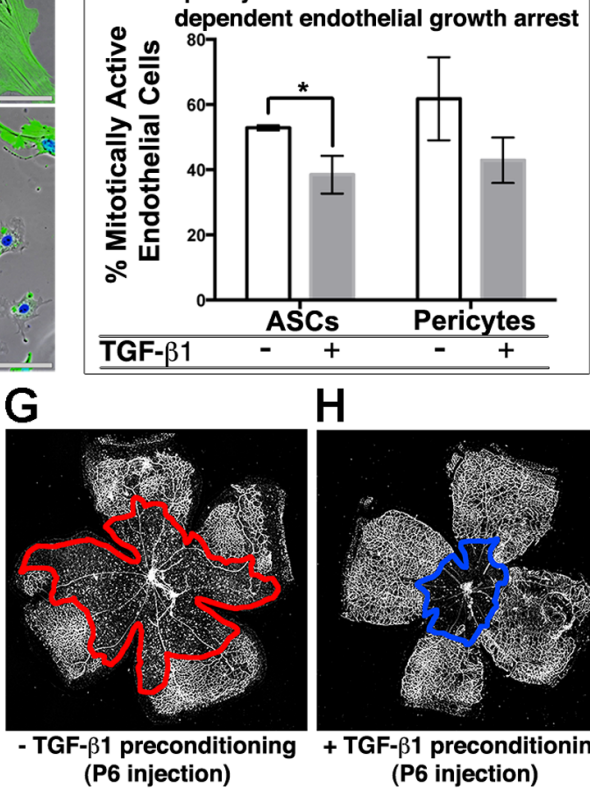

$\mathrm{H}$

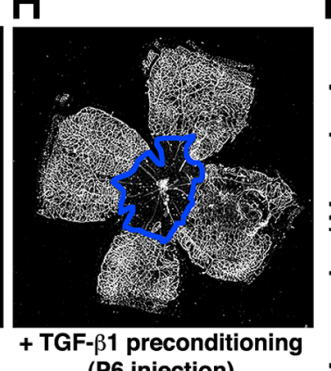

(P6 injection)
C

Contracting hASC, wrinking underlying silicone wafer

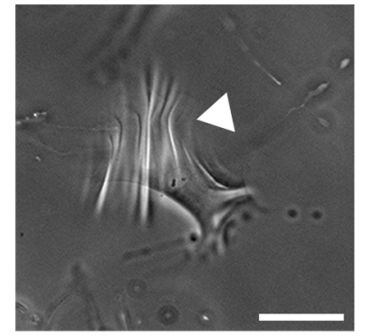

D

TGF- $\beta 1$ enhances hASC contractility
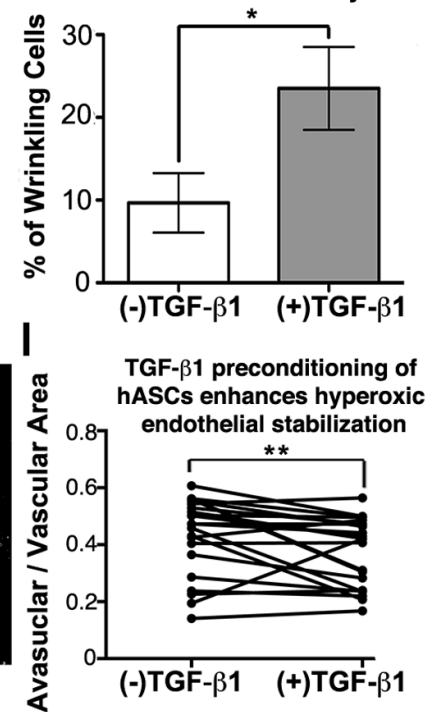

Figure 3. hASC-pericytes respond to TGF- $\beta 1$ treatment in a manner analogous to native retinal pericytes. A, B, TGF- $\beta 1 \tau \rho \varepsilon \alpha \tau \mu \varepsilon v \tau$ of endothelial monoculture and pre-conditioning of hASC/pericyte co-cultures (right panels) are compared to control serum conditions (left panels). Endothelial cells demonstrate a $23.1 \%$ decrease in S-phase nuclei, or mitotic entry (pink nuclei), when treated with TGF- $\beta 1$ alone ( $p<0.01$ ). Further, endothelial cells in direct contact (asterisks) with hASCs and pericytes (labeled green with SMA antibodies) demonstrate a $14.4 \%$ and $15.7 \%$ decrease, respectively, in cell cycle entry when hASC/pericytes are pre-treated with TGF- $\beta 1(\pi<0.05)$. C, D, TGF- $\beta 1 \pi \rho \varepsilon-\tau \rho \varepsilon \alpha \tau \mu \varepsilon v \tau$ significantly strengthens pericyte-like contractile phenotype as measured by hASC ability to deform underlying silicone substrates (white arrowhead) by 2.6 fold $(n=32$ treated cells and 40 untreated cells, $p=0.0413$ ), a response analogous to that seen with bovine retinal pericytes [53]. E, F, 48-hour pre-treatment of cultured hASCs with TGF- $\beta 1$ increases the percentage of hASCs highly expressing SMA by $15.3 \%$, without altering $\beta$ actin expression. G-I, Eyes intravitreally injected with TGF- $\beta 1$ treated $\mathrm{hASCs}$ at P6, demonstrated an $11.0 \%(n=22, p=0.01)$ reduction in retinal avascular area upon removal from hyperoxia at P12, as compared to contralateral eyes injected with untreated hASCs. Scale bars: A, C $=100 \mu \mathrm{m}$. doi:10.1371/journal.pone.0065691.g003 
The mechanism for TGF- $\beta 1$ functional improvement of vessel stabilization remains unclear. The most straightforward explanation is that TGF- $\beta 1$ pre-treatment enhances the pericyte functionality of our hASC-pericytes in a manner analogous to its effects on endogenous retinal pericytes. An alternative possibility, but one less directly supported by these results, is that our heterogeneous hASCs contain a subpopulation of pericyte precursor cells, which can be selected or functionally conditioned for endothelial protection and microvascular stabilization with TGF- $\beta 1$.

\section{ASCs Prevent Retinal Capillary Dropout in the Akimba Model of DR}

Having established that a functional retinal pericyte phenotype could be derived from hASCs, we next sought to examine their potential application for treatment of diabetic retinopathy. The recently published Akimba model features large-scale retinal capillary dropout, vessel constriction, edema, fibrosis, and bleeding in an immunocompetent diabetic mouse [35]. These findings demonstrate that this animal model replicates many more of the characteristics of severe human DR than other available models. Briefly, diabetic Ins $2^{\text {Akita }}$ mice were crossed with Kimba mice that express human VEGF165 under control of the rhodopsin promoter. The resultant Akimba pups demonstrated characteristic early onset hyperglycemia, followed by substantial weight loss and retinopathy, exhibiting profound retinal capillary dropout by 2 months of age. Because Akimba mice are fully immunocompetent, we used allogeneic murine adipose-derived stem cells (mASCs) derived from the same background strain.

We first verified that mASCs, isolated from the epididymal fat pad and cultured in the same conditions as hASCs, displayed characteristic pericyte markers. As expected, mASCs were found to express abundant steady state protein levels characteristic of native microvascular pericytes, including SMA and PDGFR- $\beta$ as measured by both flow cytometry and immunohistochemistry (Fig. 4A-C). That $14.9 \%$ of mASCis were positively stained with PDGFR- $\beta$ is consistent with similar findings in rat [56]. When injected at 5 weeks of age and harvested at 9 weeks of age, we found $\mathrm{mASC}$ s readily integrate and associate with Akimba retinal microvasculature (Fig. 4N, O).

We next tested the hypothesis that mASGs can prevent diabetic retinal microvascular dropout by injecting DiI-labeled, TGF- $\beta 1$ pre-conditioned, mASCs into the vitreous of P9 Akimba pup eyes. As before, PBS was injected as a carrier control in the contralateral eye. Two months after injection, and subsequent verification that each mouse was diabetic, retinae were harvested and retinal microvessels stained with lectin as before. Over the course of two months, no teratoma formation was observed in any of the 18 dissected eyes. Wholemount analysis of retinal vessels revealed a $79 \%$ reduction in the area of capillary dropout in retinas treated with $\mathrm{mASC}$ compared to contralateral PBS injected controls $(\mathrm{n}=9, \mathrm{p}=0.012)$ (Fig. 4D-F). Complimentary analysis of total vessel area showed a $16 \%$ reduction in absolute loss of retinal vasculature in $\mathrm{mASC}$-injected compared to contralateral PBS control-injected eyes ( $\mathrm{n}=9, \mathrm{p}=0.047$ - paired t-test) (Fig. 4G-I). Despite this dramatic reduction in vascular dropout, we interestingly found no significant reduction in the number of microaneurysms in mASC injected compared to PBS injected eyes $(n=9$, $\mathrm{p}=0.43-$ paired t-test). However, given the low frequency of microaneurysms in both cell injected and control eyes, greater numbers of animals may be needed to demonstrate a significant difference.

Prior to retinal harvest, mice were also imaged in vivo using fluorescein angiography with customized Cantor Nissel contact lenses and a Heidelberg Spectralis Retinal Imager (Fig. 4L, M). Importantly, we found that protected retinal microvessels in the mASC-injected eyes remain functional, as assessed by comparing the microvasculature by topical wholemount lectin staining (Fig. 4J, $\mathrm{K}$ ) with the corresponding fluorescein angiography (Fig. 4L, M). Taken together, these results suggest that mASCs display a pericyte phenotype analogous to hASGs and show a similar capacity to protect retinal microvessels functionally from loss in the setting of diabetic retinopathy.

\section{Conclusions}

We have demonstrated the ability of ASCs to differentiate into pericytes, as determined by pericyte-specific marker expression, in vitro confirmation of perciyte function, and response to TGF- $\beta 1$ treatment that is analogous to that of endogenous retinal pericytes. When injected intravitreally, ASCs enhance retinal microvascular stabilization in three independent pre-clinical murine models of retinopathic vasculopathy. Specifically, ASC-derived pericytes: 1 . When injected prior to OIR, protect the retinal vasculature against central vascular dropout, 2. When injected post-OIR insult, accelerate vascular regrowth and recovery of the central retinal vasculature [36], and 3. When injected at early postnatal time points, protect against capillary dropout in the Akimba murine model of DR [35]. Ex vivo treatment of ASC-derived pericyte progenitors with TGF- $\beta 1$ recapitulates the response of endogenous retinal pericytes to TGF- $\beta 1$, with enhanced pericyte marker expression, improved performance in in vitro functional assays and enhanced in vivo protection of the retinal microvasculature.

There has been increasing recognition of the role that dysregulation between pericytes and endothelial cells plays in the susceptibility of the retinal vasculature to aberrant angiogenesis and vasculopathies such as DR, age-related macular degeneration, and ROP [7,52]. DR, a disease that proceeds over many years from initial pericyte dysfunction to microvascular degeneration, may be particularly well suited for cell-based therapies. However, in the absence of a readily available supply of human retinal pericytes for transplantation, identifying a plentiful, surgically accessible, and potentially autologous alternative could offer great therapeutic potential.

Our findings demonstrate that ASGs can differentiate into pericytes and, when exogenously injected, will incorporate within the retinal microcirculation with maintained pericyte marker expression, recapitulating results using these cells in other nonocular model systems [37]. Our studies also help establish that injection of an exogenous pericyte progenitor population can functionally protect retinal microvessels against profound capillary dropout in a novel pre-clinical model of retinal vasculopathy. Given that ASCs are perivascularly located and thought to function in adipose vascular support [57], it is perhaps not surprising that they would be able to incorporate and affect the retinal vasculature in similar manner, though our studies are importantly the first direct demonstration of this.

Further work is clearly needed to illuminate the dynamic control of retinal vessel formation and degeneration in retinal vasculopathies, such as DR and ROP, especially as it pertains to the observed functional effects of injected ASCs. Exogenous insulinlike growth factor-1, along with other paracrine signals, has been shown to modify retinal vascular stabilization in OIR [58]. ASCs readily secrete insulin-like growth factor-1, more so than other types of human mesenchymal stem cells [59], and might be one method by which ASGs exert their influence on retinal vessels. Functional stabilization of retinal vasculature by ASCs, even in areas of the retina where no direct incorporation of ASC progenitors on retinal vessels is observed, lends support to the 

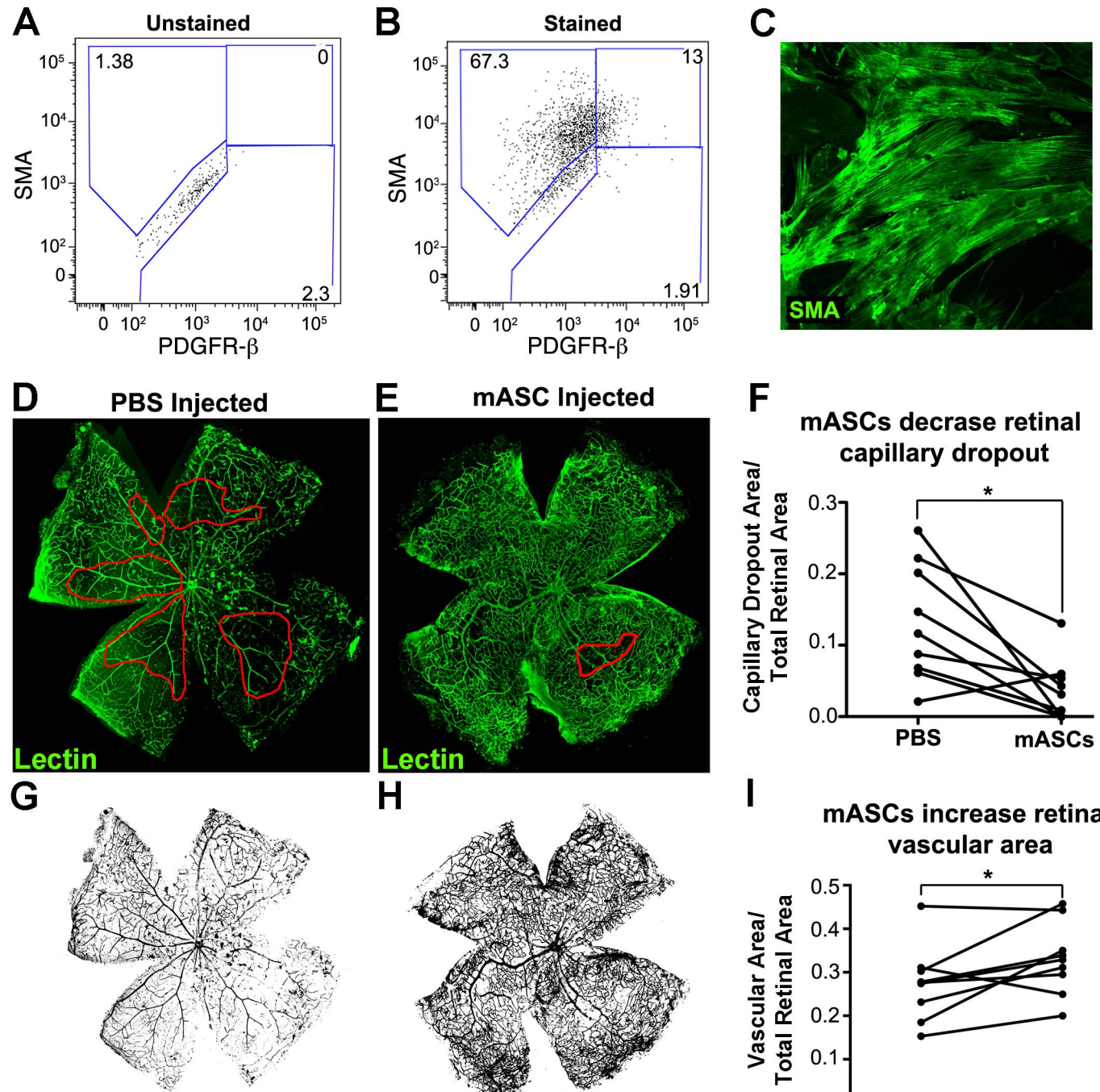

H
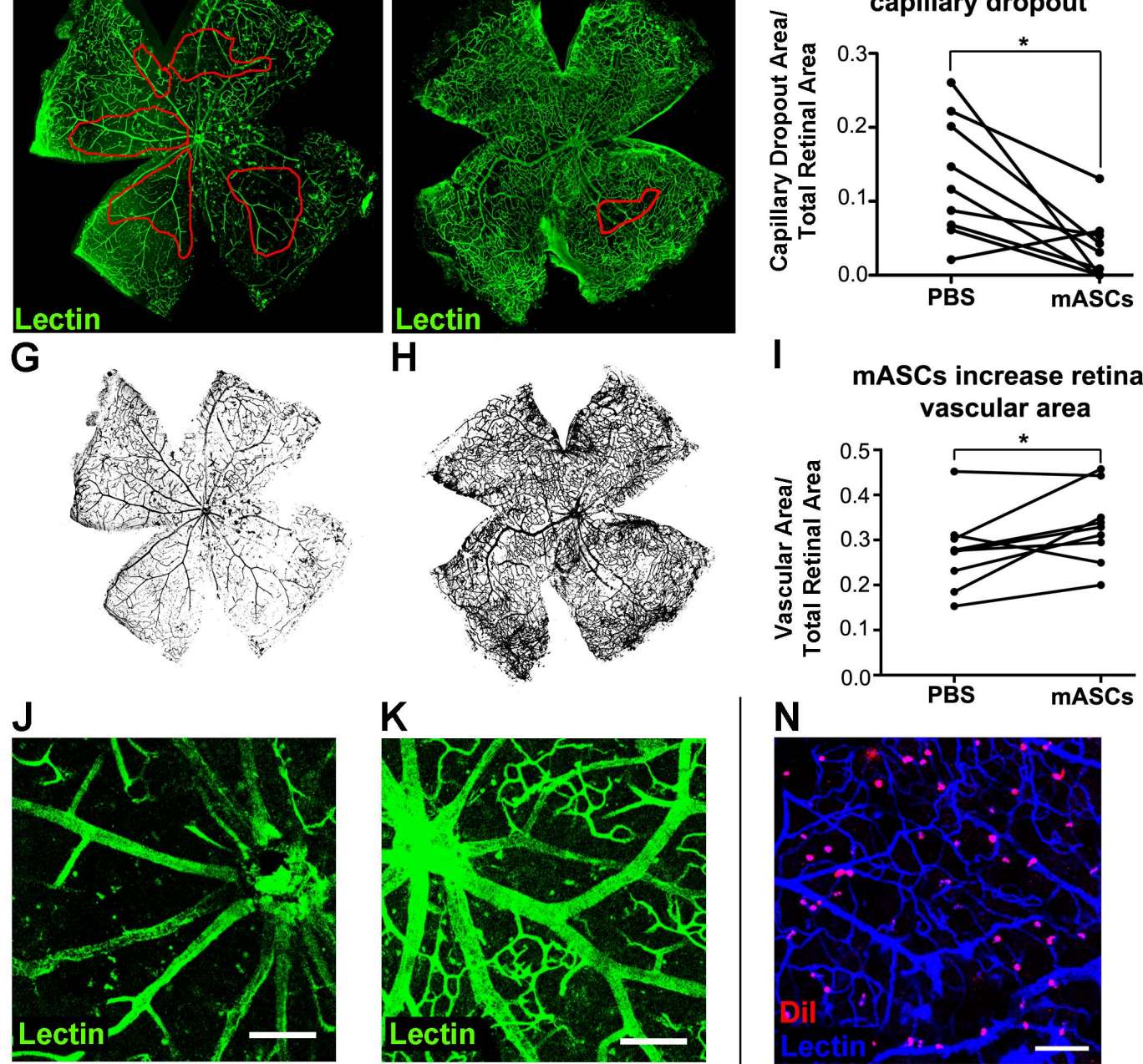

I

mASCs increase retinal vascular area
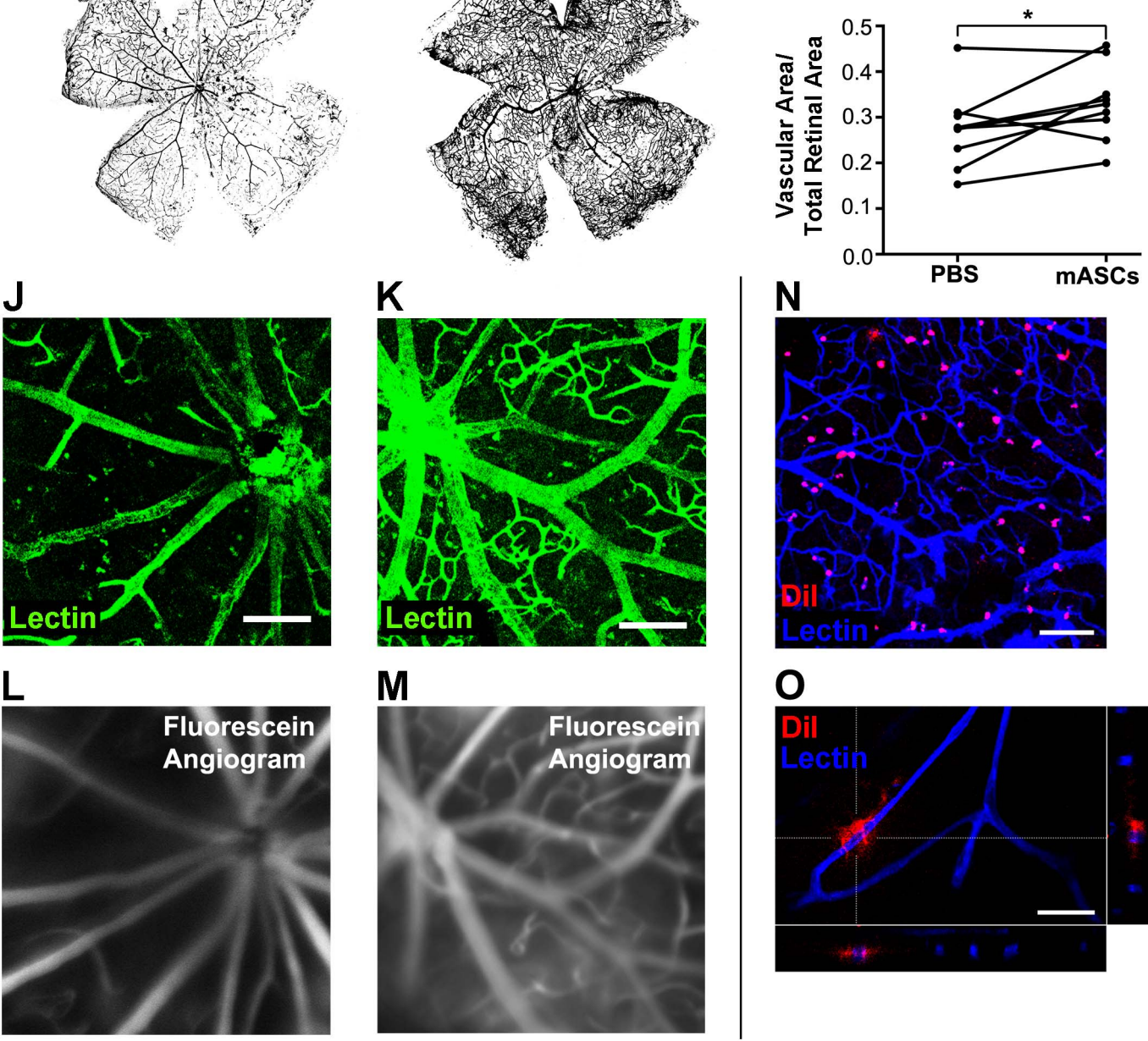

M

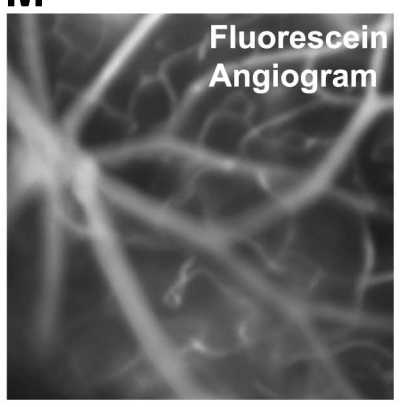

$\mathbf{N}$

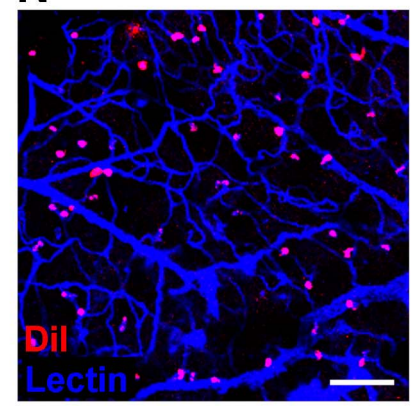

0

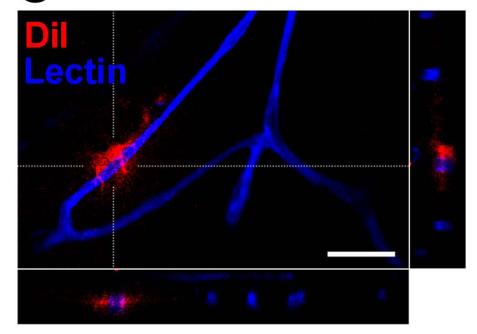


Figure 4. TGF- $\beta 1$ treated mASCs prevent long-term retinal capillary dropout in diabetic retinopathic Akimba mice. A, B, Passage 4 mouse adipose-derived stem cells (mASCs) were found to express SMA (80.3\%), PDGFR- $\beta$ (14.9\%), or both (13\%) compared to unstained controls. C, mASCs also express SMA in a typical pattern in vitro, similar to hASCs. D-M, TGF- $\beta 1$ treated mASCs were injected at P9 in Akimba mice, with PBS carrier control injected in the contralateral eye. Compared to eyes injected with PBS carrier control (D), circumscribed area of retinal capillary loss in 8 week old Akimba mice was substantially decreased in eyes injected with $\mathrm{mASCs}(\mathbf{E})$, as indicated by a $79 \%$ reduction of capillary dropout area $(\mathbf{F}$, $\mathrm{n}=9, \mathrm{p}=0.01$ ). Similarly, PBS-injected eyes (G) demonstrated an $18 \%$ lower vascular area/total retinal area ratio than contralateral mASC-injected eyes $(\mathbf{H}),(\mathbf{I}, n=9, p=0.05)$. Preservation of retinal capillaries is revealed at higher magnification by both lectin-stained retinal whole mounts $(\mathbf{J}, \mathbf{K})$ and in vivo fluorescein angiography $(\mathbf{L}, \mathbf{M})$. Note that images $\mathbf{J}-\mathbf{M}$ were taken from the same mouse. $\mathbf{N}$, After injection with Dil labeled mASCs at 5 weeks of age, subsequent harvest at 9 weeks revealed substantial integration of mASCs into an Akimba retina. $\mathbf{O}$, Di-I labeled mASC wrapping around Akimba retinal microvessel. XZ and YZ planes are also displayed. Scale bars: $\mathbf{J}, \mathbf{K}=150 \mu \mathrm{m}, \mathbf{N}=100 \mu \mathrm{m}, \mathbf{O}=25 \mu \mathrm{m}$.

doi:10.1371/journal.pone.0065691.g004

idea that ASGs exert their effects, at least in part, by conditioning the retinal microenvironment. If verified, this mechanism of action would bode well for future translational applications. It would suggest that encapsulated cells injected into the vitreous might be sufficient to stabilize retinal vessels, without requiring direct vascular integration $[42,60]$.

Additionally and importantly, recent work from our group has revealed that pericyte dysfunction, rather than pericyte loss, may be 'rate-limiting' in determining whether retinal endothelial growth and microvascular proliferative disorders ensue as outcomes or complications accompanying conditions such as diabetes $[25,28]$. In light of these findings, we intend to explore further the extent to which injected ASCs may help maintain normal function of the native pericyte population in the face of toxic environments, such as chronic hyperglycemia. Given ASC's ability to survive the relatively greater hypoxic conditions of adipose tissue as compared to the eye, they may be particularly well suited to respond to such insults.

Despite the remaining work required to translate ASC stem cell therapy to the clinic, our results suggest that stem cell based strategies for retinal vasculopathies may one day allow a shift in focus from late destructive laser treatment of hypoxic retina to earlier preventative interventions aimed at stabilizing existing retinal microvasculature. Our present findings suggest this may be accomplished using ASCis through both direct contact with retinal microvessels, as well as more general paracrine conditioning of the retinal microenvironment, both of which can prevent vessel loss and retinal hypoxia from occurring in the first place. The consistent and robust microvascular stabilizing properties of ASC-derived pericytes offer hope that such a regenerative treatment for retinal vasculopathies, including diabetic retinopathy, may be attainable.

\section{References}

1. Mario SP (2010) Global Data on Visual Impairments. World Health Organization.

2. Wong T, Yau J, Rogers S, Kawasaki R, Lamoureux EL, et al. (2011) Global Prevalence of Diabetic Retinopathy: Pooled Data from Population Studies from the United States, Australia, Europe and Asia. Association for Research in Vision and Ophthalmology Annual Meeting. Ft. Lauderdale, FL, USA.

3. Perdiguero EG, Galaup A, Durand M, Teillon J, Philippe J, et al. (2011) Alteration of developmental and pathological retinal angiogenesis in angptl4deficient mice. J Biol Chem 286: 36841-36851.

4. Cogan DG, Toussaint D, Kuwabara T (1961) Retinal vascular patterns. IV. Diabetic retinopathy. Archives of Ophthalmology 66: 366-378.

5. Romeo G, Liu WH, Asnaghi V, Kern TS, Lorenzi M (2002) Activation of nuclear factor-kappaB induced by diabetes and high glucose regulates a proapoptotic program in retinal pericytes. Diabetes 51: 2241-2248.

6. Chan-Ling T, Page MP, Gardiner T, Baxter L, Rosinova E, et al. (2004) Desmin ensheathment ratio as an indicator of vessel stability: evidence in normal development and in retinopathy of prematurity. Am J Pathol 165: 1301-1313.

\section{Supporting Information}

Figure S1 hBMSGs fail to accelerate hypoxic revascularization. Compared to contralateral PBS injected controls $(\mathbf{A})$, eyes injected with unsorted hBMSGs $(\mathbf{B})$ at P12 and harvested at $\mathrm{P} 14$ showed no statistical difference in rate of revascularization of the central retina at P14 (G). However, when compared to PBS contralateral controls $(\mathbf{D})$, eyes injected with hASGs again demonstrated a statistically significant decrease in central retinal capillary dropout $(\mathbf{F}, 16.4 \%$ reduction, $\mathrm{p}=0.03, \mathrm{n}=5)$. (TIF)

Figure S2 P2 hASG injections do not impact normal retinal vascularizaition. Compared to contralateral $P B S$ injected controls $(\mathbf{A})$, eyes injected with hASCs $(\mathbf{B})$ at P2 and harvested at P7 (without exposure to hyperoxia) displayed no obvious differences in vascular coverage, density, or morphology. (TIF)

\section{Acknowledgments}

We would like thank Anthony Bruce for his assistance with Akimba colony management; Joseph Walpole for help with FACS mASC experiments; Josia David Peske for help with FACS and subsequent FACS data analysis; Dr. Marcia McDuffie and Dr. Susanna Keller for their helpful guidance on diabetic mouse modeling; Alan Lyon for assistance with fluorescein angiography; Hulan Shang for training in hASC isolation techniques; Jeffrey Corwin for use of the confocal microscope; the UVA Flow Cytometry Core Facility, UVA Research Histology Core, and the UVA Advanced Microscopy Facility for their technical guidance.

\section{Author Contributions}

Conceived and designed the experiments: TAM EBDC TND JTD BCZ SAS SMC IMH SMP PAY. Performed the experiments: TAM EBDC DSK TND JTD BCZ SAS SMC. Analyzed the data: TAM EBDC TND JTD BCZ SAS SMC IMH SMP PAY. Contributed reagents/materials/ analysis tools: EPR AJK IMH. Wrote the paper: TAM EBDC TND JTD IMH SMP PAY.

7. Hughes S, Gardiner T, Baxter L, Chan-Ling T (2007) Changes in pericytes and smooth muscle cells in the kitten model of retinopathy of prematurity: implications for plus disease. Invest Ophthalmol Vis Sci 48: 1368-1379.

8. Herman IM, D'Amore PA (1985) Microvascular pericytes contain muscle and nonmuscle actins. J Cell Biol 101: 43-52.

9. Armulik A, Genove G, Mae M, Nisancioglu MH, Wallgard E, et al. (2010) Pericytes regulate the blood-brain barrier. Nature 468: 557-561.

10. Jechlinger M, Sommer A, Moriggl R, Seither P, Kraut N, et al. (2006) Autocrine PDGFR signaling promotes mammary cancer metastasis. J Clin Invest 116 : $1561-1570$.

11. Chen CW, Montelatici E, Crisan M, Corselli M, Huard J, et al. (2009) Perivascular multi-lineage progenitor cells in human organs: regenerative units, cytokine sources or both? Cytokine Growth Factor Rev 20: 429-434.

12. Hammes HP, Lin J, Renner O, Shani M, Lundqvist A, et al. (2002) Pericytes and the pathogenesis of diabetic retinopathy. Diabetes 51: 3107-3112.

13. Otani A, Kinder K, Ewalt K, Otero FJ, Schimmel P, et al. (2002) Bone marrowderived stem cells target retinal astrocytes and can promote or inhibit retinal angiogenesis. Nat Med 8: 1004-1010. 
14. Ritter MR, Banin E, Moreno SK, Aguilar E, Dorrell MI, et al. (2006) Myeloid progenitors differentiate into microglia and promote vascular repair in a model of ischemic retinopathy. J Clin Invest 116: 3266-3276.

15. Grant MB, May WS, Caballero S, Brown GA, Guthrie SM, et al. (2002) Adult hematopoietic stem cells provide functional hemangioblast activity during retinal neovascularization. Nat Med 8: 607-612.

16. Sengupta N, Caballero S, Mames RN, Butler JM, Scott EW, et al. (2003) The role of adult bone marrow-derived stem cells in choroidal neovascularization. Invest Ophthalmol Vis Sci 44: 4908-4913.

17. Kicic A, Shen WY, Wilson AS, Constable IJ, Robertson T, et al. (2003) Differentiation of marrow stromal cells into photoreceptors in the rat eye. J Neurosci 23: 7742-7749.

18. Kielczewski JL, Hu P, Shaw LG, Li Calzi S, Mames RN, et al. (2011) Novel protective properties of IGFBP-3 result in enhanced pericyte ensheathment, reduced microglial activation, increased microglial apoptosis, and neuronal protection after ischemic retinal injury. Am J Pathol 178: 1517-1528.

19. Chan-Ling T, Baxter L, Afzal A, Sengupta N, Caballero S, et al. (2006) Hematopoietic stem cells provide repair functions after laser-induced Bruch's membrane rupture model of choroidal neovascularization. Am J Pathol 168: 1031-1044.

20. Zuk PA, Zhu M, Mizuno H, Huang J, Futrell JW, et al. (2001) Multilineage cells from human adipose tissue: implications for cell-based therapies. Tissue Eng 7: 211-228.

21. Ding L, Saunders TL, Enikolopov G, Morrison SJ (2012) Endothelial and perivascular cells maintain haematopoietic stem cells. Nature 481: 457-462.

22. Katz AJ, Tholpady A, Tholpady SS, Shang H, Ogle RC (2005) Cell surface and transcriptional characterization of human adipose-derived adherent stromal (hADAS) cells. Stem Cells 23: 412-423.

23. Healy AM, Herman IM (1992) Density-dependent accumulation of basic fibroblast growth factor in the subendothelial matrix. Eur J Cell Biol 59: 56-67.

24. Newcomb PM, Herman IM (1993) Pericyte growth and contractile phenotype: modulation by endothelial-synthesized matrix and comparison with aortic smooth muscle. J Cell Physiol 155: 385-393.

25. Kutcher ME, Kolyada AY, Surks HK, Herman IM (2007) Pericyte Rho GTPase mediates both pericyte contractile phenotype and capillary endothelial growth state. Am J Pathol 171: 693-701.

26. Zuk PA, Zhu M, Ashjian P, De Ugarte DA, Huang JI, et al. (2002) Human adipose tissue is a source of multipotent stem cells. Mol Biol Cell 13: 4279-4295.

27. Yoshimura K, Sato K, Aoi N, Kurita M, Hirohi T, et al, (2008) Cell-assisted lipotransfer for cosmetic breast augmentation: supportive use of adipose-derived stem/stromal cells. Aesthetic Plast Surg 32: 48-55; discussion 56-47.

28. Kotecki M, Zeiger AS, Van Vliet KJ, Herman IM (2010) Calpain- and talindependent control of microvascular pericyte contractility and cellular stiffness. Microvasc Res 80: 339-348.

29. Lu F, Li J, Gao J, Ogawa R, Ou G, et al. (2009) Improvement of the survival of human autologous fat transplantation by using VEGF-transfected adiposederived stem cells. Plast Reconstr Surg 124: 1437-1446.

30. Lu F, Mizuno H, Uysal CA, Cai X, Ogawa R, et al. (2008) Improved viability of random pattern skin flaps through the use of adipose-derived stem cells. Plast Reconstr Surg 121: 50-58.

31. Huang SD, Lu FL, Xu XY, Liu XH, Zhao XX, et al. (2006) Transplantation of angiogenin-overexpressing mesenchymal stem cells synergistically augments cardiac function in a porcine model of chronic ischemia. J Thorac Cardiovasc Surg 132: 1329-1338.

32. Gruh I, Beilner J, Blomer U, Schmiedl A, Schmidt-Richter I, et al. (2006) No evidence of transdifferentiation of human endothelial progenitor cells into cardiomyocytes after coculture with neonatal rat cardiomyocytes. Circulation 113: $1326-1334$

33. Hauger O, Frost EE, van Heeswijk R, Deminiere C, Xue R, et al. (2006) MR evaluation of the glomerular homing of magnetically labeled mesenchymal stem cells in a rat model of nephropathy. Radiology 238: 200-210.

34. Cetrulo CL, Jr., Knox KR, Brown DJ, Ashinoff RL, Dobryansky M, et al. (2005) Stem cells and distraction osteogenesis: endothelial progenitor cells home to the ischemic generate in activation and consolidation. Plast Reconstr Surg 116: 1053-1064; discussion 1065-1057.

35. Rakoczy EP, Ali Rahman IS, Binz N, Li CR, Vagaja NN, et al. (2010) Characterization of a mouse model of hyperglycemia and retinal neovascularization. Am J Pathol 177: 2659-2670.

36. Smith LE, Wesolowski E, McLellan A, Kostyk SK, D’Amato R, et al. (1994) Oxygen-induced retinopathy in the mouse. Invest Ophthalmol Vis Sci 35: 101111 .

37. Amos PJ, Shang H, Bailey AM, Taylor A, Katz AJ, et al. (2008) IFATS Series: The Role of Human Adipose-Derived Stromal Cells in Inflammatory Microvascular Remodeling and Evidence of a Perivascular Phenotype. Stem Cells.
38. McVicar CM, Hamilton R, Colhoun LM, Gardiner TA, Brines M, et al. (2011) Intervention with an erythropoietin-derived peptide protects against neuroglial and vascular degeneration during diabetic retinopathy. Diabetes 60: 2995-3005.

39. Al-Shabrawey M, Mussell R, Kahook K, Tawfik A, Eladl M, et al. (2011) Increased expression and activity of 12-lipoxygenase in oxygen-induced ischemic retinopathy and proliferative diabetic retinopathy: implications in retinal neovascularization. Diabetes 60: 614-624.

40. Stitt AW, McGoldrick C, Rice-McCaldin A, McCance DR, Glenn JV, et al. (2005) Impaired retinal angiogenesis in diabetes: role of advanced glycation end products and galectin-3. Diabetes 54: 785-794.

41. Zhang SX, Ma JX, Sima J, Chen Y, Hu MS, et al. (2005) Genetic difference in susceptibility to the blood-retina barrier breakdown in diabetes and oxygeninduced retinopathy. Am J Pathol 166: 313-321.

42. Hou D, Youssef EA, Brinton TJ, Zhang P, Rogers P, et al. (2005) Radiolabeled cell distribution after intramyocardial, intracoronary, and interstitial retrograde coronary venous delivery: implications for current clinical trials. Circulation 112: I150-156

43. Guillemin GJ, Brew BJ (2004) Microglia, macrophages, perivascular macrophages, and pericytes: a review of function and identification. J Leukoc Biol 75: 388-397.

44. Hamzah J, Jugold M, Kiessling F, Rigby P, Manzur M, et al. (2008) Vascular normalization in Rgs5-deficient tumours promotes immune destruction. Nature 453: 410-414.

45. MacIsaac ZM, Shang H, Agrawal H, Yang N, Parker A, et al. (2012) Long-term in-vivo tumorigenic assessment of human culture-expanded adipose stromal/ stem cells. Exp Cell Res 318: 416-423.

46. Chan RK, Garfein E, Gigante PR, Liu P, Agha RA, et al. (2007) Side population hematopoietic stem cells promote wound healing in diabetic mice. Plast Reconstr Surg 120: 407-411; discussion 412-403.

47. Cheng LN, Duan XH, Zhong XM, Guo RM, Zhang F, et al. (2011) Transplanted neural stem cells promote nerve regeneration in acute peripheral nerve traction injury: assessment using MRI. AJR Am J Roentgenol 196: 13811387.

48. Kendirci M, Trost L, Bakondi B, Whitney MJ, Hellstrom WJ, et al. (2010) Transplantation of nonhematopoietic adult bone marrow stem/progenitor cells isolated by p75 nerve growth factor receptor into the penis rescues erectile function in a rat model of cavernous nerve injury. J Urol 184: 1560-1566.

49. Aoki H, Hara A, Niwa M, Motohashi T, Suzuki T, et al. (2008) Transplantation of cells from eye-like structures differentiated from embryonic stem cells in vitro and in vivo regeneration of retinal ganglion-like cells. Graefes Arch Clin Exp Ophthalmol 246: 255-265.

50. Zhang SX, Ma JX (2007) Ocular neovascularization: Implication of endogenous angiogenic inhibitors and potential therapy. Progress in Retinal \& Eye Research 26: $1-37$.

51. Walshe TE, Saint-Geniez M, Maharaj AS, Sekiyama E, Maldonado AE, et al. (2009) TGF-beta is required for vascular barrier function, endothelial survival and homeostasis of the adult microvasculature. PLoS One 4: e5149.

52. Papetti M, Shujath J, Riley KN, Herman IM (2003) FGF-2 antagonizes the TGF-beta1-mediated induction of pericyte alpha-smooth muscle actin expression: a role for myf-5 and Smad-mediated signaling pathways. Invest Ophthalmol Vis Sci 44: 4994-5005.

53. Sieczkiewicz GJ, Herman IM (2003) TGF-beta 1 signaling controls retinal pericyte contractile protein expression. Microvasc Res 66: 190-196.

54. Orlidge A, D'Amore PA (1987) Inhibition of capillary endothelial cell growth by pericytes and smooth muscle cells. J Cell Biol 105: 1455-1462.

55. Sato Y, Rifkin DB (1989) Inhibition of endothelial cell movement by pericytes and smooth muscle cells: activation of a latent transforming growth factor-beta 1-like molecule by plasmin during co-culture. J Cell Biol 109: 309-315.

56. Lin G, Xin Z, Zhang H, Banie L, Wang G, et al. (2012) Identification of active and quiescent adipose vascular stromal cells. Cytotherapy 14: 240-246.

57. Traktuev DO, Merfeld-Clauss S, Li J, Kolonin M, Arap W, et al. (2008) A population of multipotent CD34-positive adipose stromal cells share pericyte and mesenchymal surface markers, reside in a periendothelial location, and stabilize endothelial networks. Circ Res 102: 77-85.

58. Heidary G, Vanderveen D, Smith LE (2009) Retinopathy of prematurity: current concepts in molecular pathogenesis. Semin Ophthalmol 24: 77-81.

59. Hsiao ST, Asgari A, Lokmic Z, Sinclair R, Dusting GJ, et al. (2012) Comparative analysis of paracrine factor expression in human adult mesenchymal stem cells derived from bone marrow, adipose, and dermal tissue. Stem Cells Dev 21: 2189-2203.

60. Zhang K, Hopkins JJ, Heier JS, Birch DG, Halperin LS, et al. (2011) Ciliary neurotrophic factor delivered by encapsulated cell intraocular implants for treatment of geographic atrophy in age-related macular degeneration. Proc Natl Acad Sci U S A 108: 6241-6245. 